\title{
Total laparoscopic hysterectomy with newly designed uterine manipulator (Halder's): Safety, efficacy and perforation prevention
}

\author{
Abhijit Halder ${ }^{1}$, Indranil Dutta ${ }^{2 *}$ \\ ${ }^{\mathbf{1}}$ Assistant Professor, ${ }^{\mathbf{A}}$ Associate Professor, Dept. of Obstetrics \& Gynaecology, ${ }^{\mathbf{1}}$ College of Medicine and JNM Hospital, Kalyani, West \\ Bengal, ${ }^{2} \mathrm{IQ}$ City Medical College, West Bengal, India \\ *Corresponding Author: Indranil Dutta \\ Email: drindranildutta@gmail.com
}

\begin{abstract}
Objective: The objective of the study is to evaluate the safety and efficacy of the new uterine manipulator (Halder's) in performing Total Laparoscopic hysterectomy (TLH) and to determine whether it prevents perforation of uterus due to forward pushing force.

Introduction: Hysterectomy is one of the most common gynaecological operation performed worldwide of which laparoscopic approach is now a days preferred alternative route compared to open abdominal hysterectomy. According to U.S. Surgical data, there is a decrease of trend of abdominal hysterectomy from $65 \%$ to $54 \%$ in favour of laparoscopic route in between 1998 to 2010. It is because laparoscopic approach is associated with shortened hospitalization and postoperative recovery times and low morbidities. But it requires specialized training and skill to perform successfully. Manipulation of uterus during TLH is a vital step to avoid inadvertent injuries of vital organs like ureter, bladder and gut. Hence the newly designed uterine manipulator (Halder's) is developed to simplify TLH with its inherent quality of ease of use and simplicity. Special feature of this manipulator is the Cervical Guard with rotational forward and backward movement which distinguishes it from the all available manipulators in the market.

Material and Methods: From March 2018 to May 2019, 246 cases were done laparoscopically following a standard and simple technique using Halder's uterine manipulator in different centres in West-Bengal of which 200 cases were shortlisted as they fulfilled the inclusion criteria's of Halder's manipulator. After introduction of Halder's uterine manipulator through cervix, the operation was performed using bipolar or ultrasound energy for coagulation and cutting the vascular pedicles and ligaments. The entire procedure was done laparoscopically and the uterus was removed vaginally. The vaginal cuff was closed by continuous suture in single layer with polyglactin material.

Results: Most common indication was Abnormal uterine bleeding due to Leiomyoma(AUB-L), the average weight of uterus was $220 \pm 95 \mathrm{gms}$. Mean operation time was $64.6 \pm 15.5$ minutes. Estimated blood loss was $80 \mathrm{ml} \pm 20 \mathrm{ml}$. There was no bowel injury but one ureteric injury, one Bladder injury and one uterine perforation were found. Great ranges of movement found during manipulation.

Conclusion: The easy, smart and simple Halder's uterine manipulator could be the option to do TLH, in reducing the complications and having more favourable operative outcomes. This manipulator can be used by inexperienced persons at vaginal end.
\end{abstract}

Keywords: Total laparoscopic hysterectomy, Quality assessment questionnaire sheet, Halder's uterine manipulator, Cervical guard, Uterocervical insert, Colpotomy tube, Colpotomy tube fixation screw.

\section{Introduction}

Hysterectomy is one of the most common gynaecological operation performed worldwide of which laparoscopic approach is now a days preferred alternative route compared to open abdominal hysterectomy. According to U.S. Surgical data, there is a decrease of trend of abdominal hysterectomy from $65 \%$ to $54 \%$ in favour of laparoscopic route in between 1998 to $2010 .{ }^{1}$ It is because laparoscopic approach is associated with shortened hospitalization and postoperative recovery times and low morbidities. But it requires specialized training and skill to perform successfully. Till today hysterectomy is being performed abdominally because of its easier learning curve compared to laparoscopic approach. ${ }^{2}$ Laparoscopic approach requires specialized trainings which is usually not available in most of the Medical Colleges. Laparoscopic approach is associated with better outcome in respect to post-operative morbidity and faster recovery and reduced long-term complications like incisional hernia, adhesion formation. ${ }^{3}$ For laparoscopic gynaecologist, one of the most important step is proper uterine mobilisation to reduce complications. ${ }^{4}$ There is a high incidence of serious complications with TLH such as ureteric injury, bladder injury, haemorrhage etc due to less experience of surgeons. ${ }^{5}$
Proper manipulation of uterus is the primary necessary step for smooth performance of Total Laparoscopic Hysterectomy. The debate stays between Myoma screw and uterine manipulator as best method for uterine manipulation, because no method is perfect for performing TLH till date. Uterine manipulator or myoma screw both has promising role in successful and uncomplicated TLH operation. Myoma screw acts by pulling principle and uterine manipulators depend on push method. Pushing method sometimes end up in perforation of uterus in the midst of operation. Myoma screw blocks one assistant port which is considered to be the main drawback. Several types of uterine manipulators have been developed to improve the TLH operation till date such as Clemont-Ferrand's, HOHL, RUMI with KOHs, Hourcabie, endopath, Vcare, Dr Mangeshikar etc. ${ }^{6,7}$ Amongst them the Clemont-Ferrand, RUMI, Dr Mangeshikar are of first line choices by surgeons. There is no single manipulator which can fulfil all the criteria to be the best manipulator. ${ }^{7,8}$ Clemont-Ferrand, RUMI with $\mathrm{KOH}$ are very expensive, and is a complex to assemble. Moreover RUMI with $\mathrm{KOH}$ has restricted elevation movement and also not applicable in narrow vagina. V CARE single use system which adds to the cost and not applicable for large uterus. Multiple reports showed 
disintegration of instrument and parts to be left behind with RUMI I, V Care, Clearview. ${ }^{8}$ Dr Mangeshikar manipulator manufactured by Karl Storz, Germany is the most popular manipulator now a days because of its less difficult assembly mechanism, unique levo and dextro rotation movement, good ante and retroversion movement, lateral movement with well pneumo-occluder function. ${ }^{9}$ Pneumoperitoneum maintainance is very essential criteria for TLH operation which is lacking in Endopath, Hourcabie.

Unusual complication like iatrogenic uterine rupture which also caused bowel perforation is reported by HOHL. ${ }^{10}$ Uterine perforation by CLEARVIEW, V CARE has also been reported in MAUDE database and literature. ${ }^{6,9}$ The intrauterine tip of $\mathrm{V}$ CARE perforated the uterus whereas perforation during cervical dilation before installation has been reported with CLEARVIEW. ${ }^{7}$ Balloon perforation has been reported with RUMI due to intrauterine balloon hyper insufflation. ${ }^{11}$ Uterine perforation during forceful manipulation makes the operation difficult and prolonged, which is underreported but often faced by the surgeons. Surgeons can manage to complete TLH with difficulty or by using Myoma screw from above. But it is definite that the operation becomes hazardous due to unnecessary bleeding from the perforation site and recurrent perforation by assistant through the same site in the midst of operation. Operation time is also prolonged which makes the surgeon irritated and impatient.

The Halder's uterine manipulator was developed to alleviate most of available uterine manipulators' drawbacks most importantly for avoiding perforation complication. This instrument has no chance of disarticulation and can maintain pneumoperitoneum well along with good manipulation and movement ranges. As it is a very low cost device and reusable, it is better suited in developing country health care environment.

This instrument was designed by a Gynaecologist of West Bengal, India who had difficulty during TLH due to perforation caused by his vaginal assistant regularly in his initial time of laparoscopic surgery. This instrument is solely based on a rod with a nut like rotating device. The concept of cervical guard on the shaft of the instrument made changes and the assistant couldn't perforate even with greatest force in which he was practiced to. Following this, the device with modification made from factory distributed to his local colleagues for use and they also got excellent results. Now this modified simple instrument is being used in West Bengal and some other centres outside West Bengal in India with the same benefits.

\section{Aims and Objectives}

\section{Primary objective}

To evaluate the efficacy and safety of the newly designed uterine manipulator 'Halder's uterine manipulator' in performing TLH.

\section{Secondary objective}

1. To determine the chance of uterine perforation by forward pushing force during manipulation.

2. To determine the ranges of movement of the manipulator and satisfaction level by laparoscopic surgeons.

\section{Inclusion and cxclusion criteria}

Patients who had benign gynaecologic diseases like adenomyosis, fibroid, endometrial hyperplasia, Grade I, II Endometriosis requiring hysterectomy were included.

Patients with Gynaecological cancers and cases with severe (Grade III, IV) endometriosis were excluded from the study.

\section{Materials and Methods}

\section{Study period}

March 2018 to May 2019.

\section{Study areas}

This prospective descriptive study was conducted at different centres in West-Bengal, like Joymala Memorial Hospital, Kalyani, Care and Cure private Hospital, Barasat, IQ City Medical College, Durgapur, Eden Hospital, North 24 Parganas, Apollo Gleneagles Hospital, Kolkata, Ruby General Hospital, Kolkata by different laparoscopic Surgeons after obtaining due consent from them to participate in the study. The surgeons of those hospitals were provided with Quality Assessment Questionnaire for feedback. The instrument was provided to them for trial in their cases and method of use was taught by YouTube video demonstration. ${ }^{12}$

\section{Study materials}

246 cases were done laparoscopically following a standard and simple technique using Halder's uterine manipulator in different centres in West-Bengal of which 200 cases were shortlisted as they fulfilled the inclusion criteria's of Halder's manipulator.

Age, weight and BMI of each patient were asked to be recorded by the surgeons. The information of total number of TLH with their indication, previous operation history, average time taken for each operation, estimated blood loss in $\mathrm{ml}$ were asked to be recorded by the surgeons in each operation.

Surgeons were provided with the Quality Assessment Questionnaire sheet to be filled up in due time. The data collected and analysed.

\section{Instrument description}

The Halder's uterine manipulator (Fig. 1) is based on a simple stainless-steel shaft (B) of $45 \mathrm{~cm}$ length. It is specially designed in a thread like fashion which has the capability of a rotating a screw nut like device which is named as cervical guard in this device.

The Accessories and parts of Halder's manipulator (marked by alphabet as below in Fig. 2, Fig. 5) 
1. 3 cervical inserts $(4 \mathrm{~cm}, 6 \mathrm{~cm}, 8 \mathrm{~cm})$

2. Shaft or Rod

3. Cervical guard with a hole $(3 \mathrm{~cm}$ outer diameter)

4. Threads at rod tip $(5.5 \mathrm{~cm}$ length $)$

5. 2 cervical cups ( $35 \& 40 \mathrm{~mm}$ in diameter) with ceramic margin

6. Colpotomy tube

7. Colpotomy tube fixation screw

8. Rod handle - detachable

The distal end of the shaft has handle $(\mathrm{H})$ which is detachable by screwing movement (Fig. 1).

The metallic Cervical Guard (C) is $3 \mathrm{~cm}$ in outer diameter and of $2 \mathrm{~cm}$ thickness with a hole of $2.8 \mathrm{~cm}$ diameter. It can be screwed over the threads (D) on the shaft (B). It can move forward and backward with clockwise and anticlockwise movement respectively. It helps to achieve more length in the utero-cervical canal while elevation movement and doesn't allow the rod to be advanced more in the uterine cavity during forceful manipulation during TLH. Even single cervical insert fitted at the shaft tip can perform various surgery of different uterocervical length because length can be changeable with rotational movement of the cervical guard forward and backward.

The uterocervical inserts (A) were $0.5 \mathrm{~cm}$ breadth and come in 3 different length $(4 \mathrm{~cm}, 6 \mathrm{~cm}, 8 \mathrm{~cm})$ for different size uterus (Fig. 2, 5).

Each has an upward 15 degrees angle for anteverted and retroverted uterus and can be connected to the shaft tip. After measuring the utero-cervical length, appropriate insert can be fit at the tip of the instrument. The tubular cervical cups (E) were made of ceramic material $(20 \mathrm{~mm})$ at proximal edge. Each was $6 \mathrm{~cm}$ in height and came in 2 different diameters of 35 and $40 \mathrm{~mm}$ respectively.

The Colpotomy Tube (F) can be attached with different sizes of colpotomy cup (E). It has a Fixation Screw (G) which is used to fix the colpotomy tube with cup to be fixed anywhere over the shaft of the instrument. The Handle $(\mathrm{H})$ is also detachable by clockwise rotational movement from the shaft. This feature helps the vaginal assistant to introduce the colpotomy cup any time in the middle of operation without exteriorising the whole instrument.

The diagram of Halder's manipulator as in patent application showing all parameters is given in Fig. 5. The patent application is published by Government of India (Fig. $6)$.

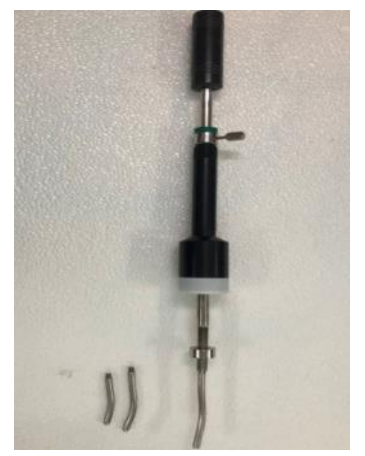

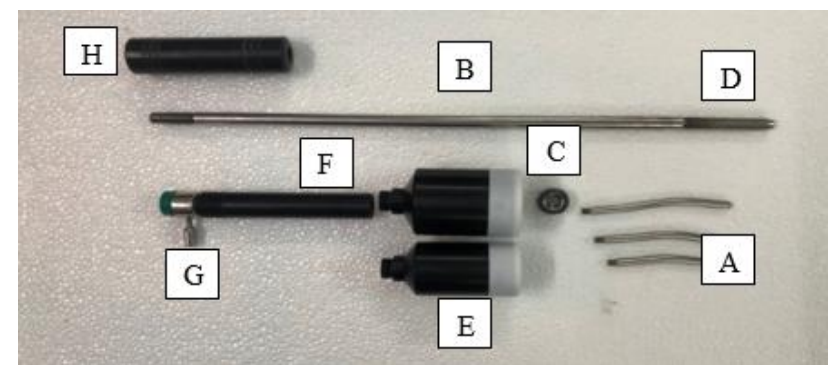

Fig. 2

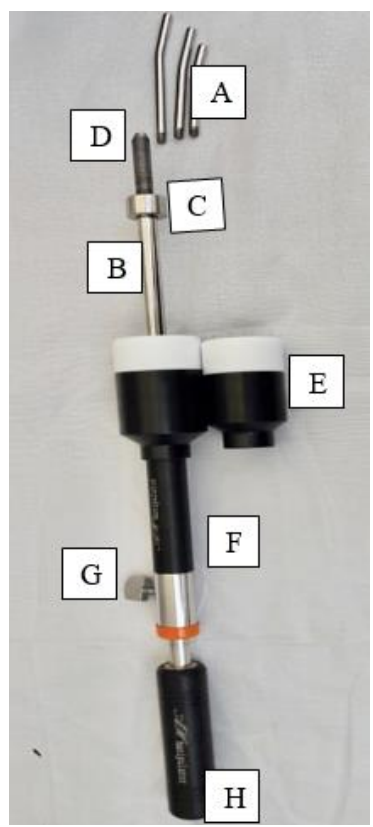

Fig. 3

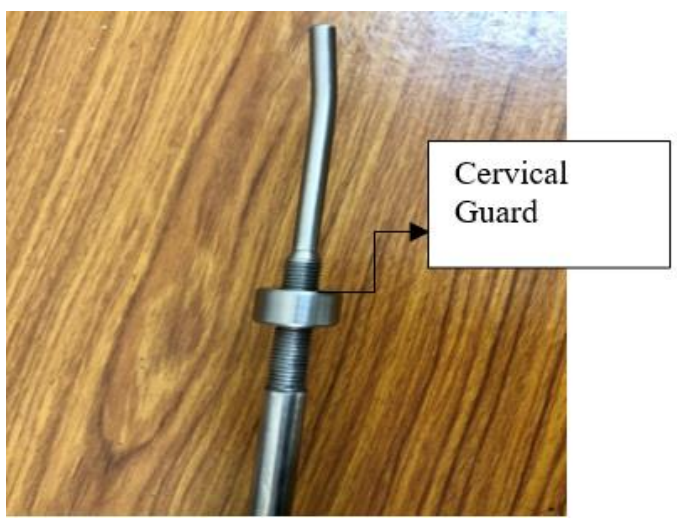

Fig. 4



Fig. 5

Fig. 1 


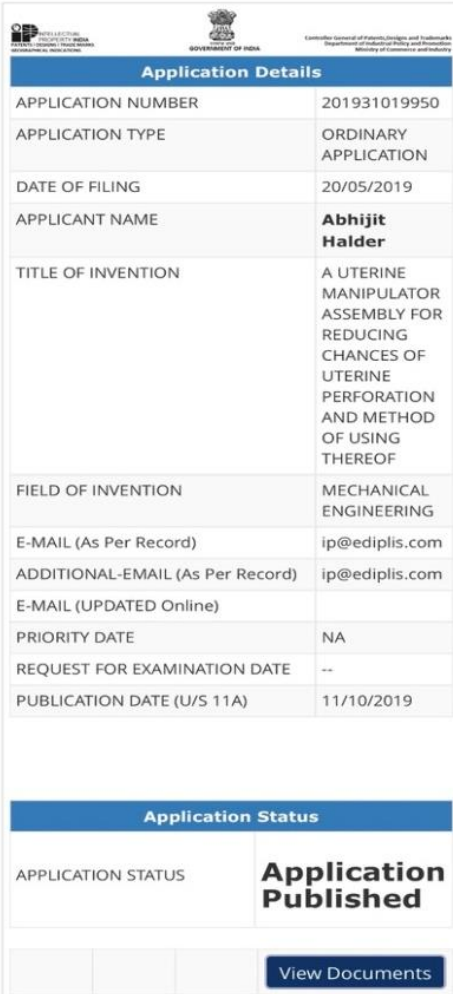

Fig. 6: Patent document

\section{Installation technique of Halder's manipulator}

After putting patient on general anaesthesia and positioning her in lithotomy position with the buttocks at the edge of the operation table, uterine mobility, size and position were assessed by vaginal examination. It will help to get idea about appropriate cup size to be taken. All Halder's uterine manipulator components were sterilized prior to each operation. The patient's bladder was then emptied and the catheter retained.

After the cervical canal was dilated with the Hegar's dilator, upto $5 \mathrm{~mm}$. the appropriate length insert (A) is selected by measuring the uterocervical canal by uterine sound or by the Hegar's dilator keeping them side by side. Even if proper length not ascertained it may be directly put into the canal and the cervical guard is rotated forward or backward to get proper position so that it does not perforate during elevation of uterus forcefully. The speciality of this instrument is rotating movement of the cervical guard on the rod which makes it unique.

Following this the TLH operation is performed with or without $\mathrm{B} / \mathrm{L}$ salpingo-oophorectomy. The operation technique may differ surgeon to surgeon. The colpotomy tube with cup may be put inside the vagina from the beginning of operation or may be slid over later and fixed over the rod with the fixation screw. The surgeons who prefer to use the cup only for colpotomy, they may not use the cup with tube from beginning. When the mackenrodt's ligaments are coagulated and cut they may put the colpotomy tube with cup from behind after detaching the handle from the shaft not exteriorising the instrument from uterine cavity. It alleviates the need of repeated introduction of the instrument through the cervix. The colpotomy cup with tube may be kept already over the rod but outside the vagina (mostly done by the surgeons) until the step of Colpotomy. Even when there is difficulty to delineate the area upto which bladder needs to be dissected down over the cervix, surgeon may push the colpotomy tube (E) with cup (D) over the rod. As the diameter of the smallest cup is more than the cervical guard (Fig. 3), the colptomiser easily goes over it to be placed at the fornices over the cervix. Following this, exaggerated forward push by the assistant with the colpotomy tube makes the UV fold prominent, lateralises the ureter and more traction obtained which help in precise dissection, safe coagulation and cutting. The cup can be fixed over the shaft at this stage by fixing it with the fixation screw. It helps the assistant to use only one hand and another to rest if fatigue. Colpotomy done by monopolar current. Specimen retrival done by holding the cervix by vulsellum under camera guidance. During this step a folded mop is put over the external vaginal orifice, beside the vulsellum to prevent gas leak. Intracorporeal vault suturing was done by each surgeon. Total operating time was recorded in OT details. All patients were followed up by each surgeon after 6 weeks.

\section{Results}

From March 2018 to May 2019, total of 246 cases were done with Halder's uterine Manipulator including all the 6 hospitals by 10 different surgeons. An average of 25 surgeries with Halder's manipulator were done by each surgeon in the time period.

Patients were between the ages of 42 and 64. Their average age and BMI were 44.4 years and $23.8 \mathrm{~kg} / \mathrm{m}^{2}$. All patients underwent TLH procedure using Halder's uterine manipulator. Indications for hysterectomy were AUB-L, AUB A, Grade 1 and 2 Endometriosis and endometrial hyperplasia in $85,74,24$ and 17 patients respectively. Previous surgical history on uterus of the study population is depicted in table 1 .

Patient characteristics and the operative outcomes are shown in Table 2. The mean operating time was 64 minutes. The mean uterine size was 220 grams ranged from $70-1100$ grams. There were 1 case of bladder injury, 1 case of ureteric injury, 1 case of uterine perforation by instrument tip amongst 200 cases recruited in the study.

Table 1: History of previous surgery in TLH patients operated with Halder's uterine manipulator $(\mathrm{n}=200)$

\begin{tabular}{|l|c|}
\hline No previous operation on uterus & 74 \\
\hline Previous h/o 1 caesarean section & 38 \\
\hline Previous h/o 2 caesarean section & 18 \\
\hline Previous h/o 3 caesarean section & 2 \\
\hline Previous h/o myomectomy & 4 \\
\hline Previous h/o ectopic operation & 2 \\
\hline
\end{tabular}


Table 2: Patient characteristics and operative features

\begin{tabular}{|l|c|}
\hline Patient characteristics & Operative features \\
\hline Age (years) & $44.4 \pm 2.5$ \\
\hline Body weight $(\mathrm{kg})$ & $58.5 \pm 6.6$ \\
\hline BMI $\left(\mathrm{kg} / \mathrm{m}^{2}\right)$ & $23.8 \pm 3.5$ \\
\hline Uterine size $($ grams $)$ & $220 \pm 95$ \\
\hline Operative times $(\mathrm{min})$ & $64 \pm 15.5$ \\
\hline Estimated blood loss $(\mathrm{ml})$ & $60 \pm 20$ \\
\hline
\end{tabular}

Table 3: Intraoperative major complications in TLH with Halder's uterine manipulator assisted operation

\begin{tabular}{|l|c|c|c|c|c|c|}
\hline No of patients & $\begin{array}{c}\text { Bladder } \\
\text { injury }\end{array}$ & $\begin{array}{c}\text { Ureteric } \\
\text { injury }\end{array}$ & $\begin{array}{c}\text { Bowel } \\
\text { injury }\end{array}$ & $\begin{array}{c}\text { Uterine perforation } \\
\text { with instrument tip }\end{array}$ & $\begin{array}{c}\text { Parts retained } \\
\text { in uterine cavity }\end{array}$ & $\begin{array}{c}\text { Vescico-vaginal } \\
\text { fistulae }\end{array}$ \\
\hline 200 & 1 & 1 & 0 & 1 & 0 & 0 \\
\hline
\end{tabular}

Table 4: Efficacy and safety features found in TLH with Halder's uterine manipulator depending on Questionnaire Feedback (Total Score for each criteria-5)

\begin{tabular}{|c|c|c|c|c|c|c|c|c|c|}
\hline  & 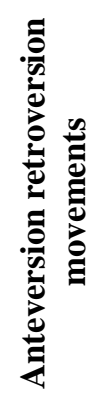 & 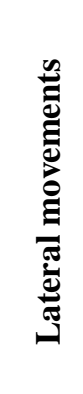 & 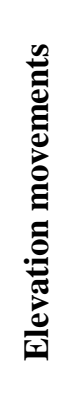 &  & 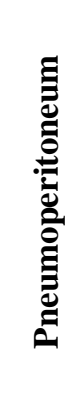 & 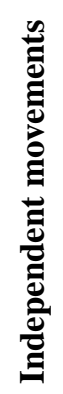 & 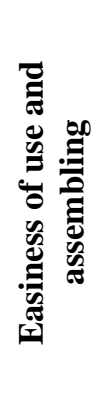 & 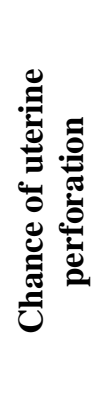 & 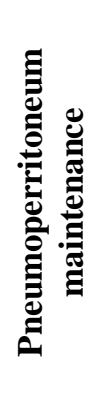 \\
\hline $\begin{array}{l}\text { Average score obtained } \\
\text { from the surgeon's feedback }\end{array}$ & 4.4 & 4.8 & 5 & 5 & 4.6 & 5 & 5 & 4.9 & 4.5 \\
\hline
\end{tabular}

Table 5: The advantages and disadvantages of Halder's Uterine Manipulator (interpreted depending on the feedback provided through the questionnaire by different surgeons)

\begin{tabular}{|c|c|}
\hline Advantages & Disadvantages \\
\hline $\begin{array}{l}\text { 1. Movement ranges }+130^{\circ} \text { in anterior plane, } 90^{\circ} \text { in the posterior } \\
\text { plane when only the rod with cervical guard used. } \\
\text { 2. With colpotomy tube pushed inside over the rod, it is } 90^{\circ} \text { in } \\
\text { anterior plane and } 45^{\circ} \text { in posterior plane. } \\
\text { 3. Reusable instrument. } \\
\text { 4. Pneumooccluder function is good } \\
\text { 5. Less costly } \\
\text { 6. Very less time needed for installation of the manipulator. } \\
\text { 7. Easily cleaned and sterilised, as less joints. } \\
\text { 8. No chance of disintegration of parts to be left behind. } \\
\text { 9. Effective in large uterus as whole uterocervical canal length } \\
\text { can be used for manipulation. } \\
\text { 10. Inexperienced vaginal assistant can perform easily on verbal } \\
\text { commands. }\end{array}$ & $\begin{array}{l}\text { 1. Specimen retrieval depends on camera and } \\
\text { vulsellum. No inherent easy technique for } \\
\text { specimen retrieval. } \\
\text { 2. In large uterus (more than } 12 \text { wks size) specialy } \\
\text { where there is posterior fibroid, myoma screw } \\
\text { was needed for better visualisation of the } \\
\text { posterior peritoneum and adnexal structures. } \\
\text { 3. No independent movement at the instrument tip, } \\
\text { levo and dextrorotation of utero-cervical insert } \\
\text { is not possible. } \\
\text { 4. External vaginal orifice is the fulcrum for the } \\
\text { leverage system of the manipulator. }\end{array}$ \\
\hline
\end{tabular}

\section{Discussion}

Uterine manipulator is an important tool which can improve outcome of TLH. ${ }^{5}$ The most dreadful complication faced till today is ureteric injury. Uterine manipulator was developed for prevention of injury to adjacent organs and it has shown promising results. ${ }^{7,8}$

A good manipulator which is user friendly and will help avoid dreadful complications, will make beginners to do more laparoscopic surgery than abdominal open surgery.

Though there are many Manipulators available in market, no single instrument is not without any drawback. Some are very good in anteversion/retroversion and lateral movements, some lack that characteristic. Few are advantageous from other aspect like pneumoperitoneum maintenance, reusability etc. 
In case of Clermont Ferrand and RUMI System, the mechanism of assembly itself is quite difficult. ${ }^{8}$

The Halder's uterine manipulator is designed to overcome most of drawbacks and difficulties of available uterine manipulators specially in the scenario of a developing country health care facility.

If only the rod with cervical guard is used with the vaginal orifice as fulcrum, ranges of movement excellent as because of its inherent quality to avoid accidental perforation of uterus. But if colpotomy tube is put inside, the range of motion decreases little bit.

The vaginal fornices can be easily identified when the colpotomy tube with cup is pushed inside over the rod. Ureter can be more lateralized from the field of surgery when the instrument is pushed forward and colpotomy tube is fixed over the rod with fixation screw. The colpotomy cup with tube act as very good pneumo-occluder. No leak of gas has been demonstrated in the study.

The drawback of this instrument is that removal of the specimen is to be done by vulsellum under camera guidance. But most of the surgeons are familiar with specimen retrieval technique through vagina. During vulsellum introduction for holding the cervix there is chance of loss of pneumo-peritoneum but this is not a problem if a folded mop is wrapped over the external vaginal orifice.

Only number 5 hegar's dilatation is necessary for Halder's Manipulator as the tip insert is of only $5 \mathrm{~mm}$ breadth. Only one assistant is enough to install the instrument. Less expertise personnel can do application and manipulation easily.

In this study it was found that we had one case of bladder injury, one ureteric injury and another case of uterine perforation amongst the 200 cases (Table 3). The patient who had bladder rent was a patient with previous 2 caesarean section. Bladder was densely adherent to the anterior uterine wall and anterior abdominal wall. There was no role of manipulator in causation of bladder injury. The ureteric injury was during cutting the ovarian pedicle. The ovary was grossly adhered to posterolateral wall of uterus and ovarian fossa. Maybe, the surgeon was unaware of the chance of ureter to be drawn up. In that particular case where uterine perforation happened was due to exaggerated dilatation of internal OS due to chronic use of norethisterone for more 3 months to control bleeding. The uterus was very soft and OS dilated more than needed due to preoperative 2 tablets of misoprostol application. As a result the cervical guard which was $3 \mathrm{~cm}$ in diameter went through the OS. It is to be mentioned that the diameter of the cervical guard has been increased to $3.5 \mathrm{~cm}$ to relatively avoid such occurrence. The surgeons have to be aware of the consistency and dilatability of the OS before operation. They shouldn't use norethisterone for long term before operation.

During search for perforation with uterine manipulator studies are scarce. Few case reports on perforation by uterine manipulators were found. One case happened by RUMI manipulator in 2005, one case with RUMI with $\mathrm{KOH}$ in 2007, 2 cases by Kronner's Manipulator in 2007 and
$2015 .^{10,11}$ Most of the perforation reported in Non TLH Gynaecological cases. It may be due to the reason that in $\mathrm{TLH}$, as the uterus anyway is to be removed, no uterine perforation was important to be reported. No study focussed on the difficulty of operation if there was perforation by the tip of the instrument in the middle of the operation. Though underreported it is quite common during TLH and often faced by the surgeon, especially if the design of the tip of the manipulator is like Hegar's Dilator. Even though the instrument tip is withdrawn from the perforation site, and directed to other area of fundus away from perforation, the instrument tip again comes out through the same perforation site during forward pushing. As a result, the assistant becomes attentive not to push forcefully to restrain from perforation. Halder's manipulator is devoid of this problem. Surgeon as well as the assistant are satisfied to each other during surgery with Halder's Manipulator.

\section{Conclusion}

Halder's Uterine Manipulator is a modification of previously available popular manipulators with the advantage of avoiding perforation complication of the uterus. It has very less articulation and joints. It only depends upon screwing rotational movements of the cervical guard, colpotomy cup and the handle. So only one type of movement has to be remembered by the vaginal assistant. It makes the instrument user friendly. Pneumoperitoneum is well maintained by this device. The device will allow beginners and less experienced surgeons to perform a successful TLH with more confidence and better outcomes. If endometriotic colon adhesions or gut adhesion over the uterus due to PID found during TLH, this instrument can be used without the fear of having iatrogenic gut or bladder injury due the presence of Cervical Guard which will guard the assistant from accidental uterine perforation even if forceful manipulation done. As learning curve is very steep it will become user friendly also. These qualities may help the Halder's Uterine Manipulator to be the instrument of choice. This instrument is cheap, smart, easy to use and effective in performing most of the cases in the field of Gynaecological surgery. These results need to be evaluated and confirmed in a larger numbers of patients.

\section{Conflict of Interest}

None.

\section{Source of Funding}

Self only for initial manufacturing of the manipulator which was reasonable and supplied to others.

\section{References}

1. Choosing the Route of Hysterectomy for Benign Disease. ACOG in collaboration with committee members, Kristen A. Matteson, Samantha F. Butts, MSCE Committee Opinion Number 701, November 2017.

2. Millar WJ. Hysterectomy, $1981 / 82$ to $1996 / 97$. Statistics Canada. Health Reports2001;12:9-22. 
3. Aarts JW, Nieboer TE, Johnson N. Surgical approach to hysterectomy for benign gynaecologicaldisease. Cochrane Database Syst Rev. 2015;(8):CD003677.

4. Albright BB, Witte T, Tofte AN. Robotic Versus Laparoscopic Hysterectomy for Benign Disease: A Systematic Review and Meta-Analysis ofRandomized Trials. J Minim Invasive Gynecol. 2016;23:18-27.

5. Wattiez A, Soriano D, Cohen SB, Nervo P, Canis M, Botchorishvili R, et al. The learning curve of total laparoscopic hysterectomy: comparative analysis of 1647 cases. J Am Assoc Gynecol Laparosc. 2002;9:339-45.

6. Koh $\mathrm{CH}$. A new technique and system for simpli- fying total laparoscopic hysterectomy. J Am Assoc Gynecol Laparosc. 1998;5:187-92.

7. Van den Haak. L, Alleblas. C. Efficacy and safety of uterine manipulators in laparoscopic surgery: A review. Arch Gynecol Obstet. 2015;015-3727-9.

8. Mettler L, Nikam YA. A comparative survey of variousuterine manipulators used in operative laparoscopy. Gynecol Surg. 2006;3:239-43

9. Hedge CV. Mangeshikar uterine manipulator. J Obstet
Gynaecol India. 2016;66(2):134-6.

10. Akdemir A, Cirpan T. Iatrogenic uterine perforation and bowel penetration using a Hohl manipulator: A case report. Int J Surg Case Rep. 2014;5:271-3.

11. Nwanodi OB, Novac S, Khulpateea N. Iatrogenic uterine perforation with Kronner uterine manipulator. Gyneacol Obstet Case Rep. 2015;1(1:2):1-4.

12. The patency application of Halder's uterine Manipulator has been published by Government of India on 20/05/2019 (Application number-201931019950).

13. YouTube video demonstration. (https://youtu.be/im7LuHQLrhU).

How to cite: Halder A, Dutta I. Total laparoscopic hysterectomy with newly designed uterine manipulator (Halder's): safety, efficacy and perforation prevention. Panacea J Med Sci. 2020;10(1):3-9. 